\title{
Radiation Hardness of the LHCb Outer Tracker
}

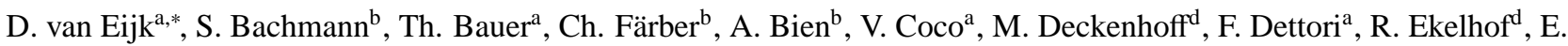

Gersabeck $^{\mathrm{b}}$, T.M. Karbach ${ }^{\mathrm{d}}$, R. Koopman ${ }^{\mathrm{a}}$, A. Kozlinskiy ${ }^{\mathrm{a}}$, Ch. Langenbruch ${ }^{\mathrm{b}}$, Ch. Linn ${ }^{\mathrm{b}}$, M. Merk ${ }^{\mathrm{a}}$, M. Meissner ${ }^{\mathrm{b}}$, P.

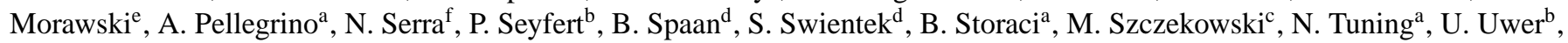
E. Visser ${ }^{\mathrm{a}}$, D. Wiedner ${ }^{\mathrm{b}}$, M. Witek ${ }^{\mathrm{e}}$

${ }^{a}$ Nikhef, Amsterdam, The Netherlands

${ }^{b}$ Physikalisches Institut, Heidelberg, Germany

${ }^{c}$ A. Soltan Institute for Nuclear Studies, Warsaw, Poland

${ }^{d}$ Technische Universität Dortmund, Germany

${ }^{e}$ H. Niewodniczanski Institute of Nuclear Physics, Cracow, Poland

${ }^{f}$ Physik-Institut, Universität Zürich, Switzerland

\begin{abstract}
This paper presents results on the radiation hardness of the LHCb Outer Tracker (OT) during LHC operation in 2010 and 2011. Modules of the OT have shown to suffer from ageing effects that lead to gain loss, after irradiation in the laboratory. Under irradiation at moderate intensities an insulating layer is formed on the anode wire of the OT straw cells. This ageing effect is caused by contamination of the counting gas due to outgassing of the glue used in the construction of the OT modules. Two methods to monitor gain stability in the OT are presented: module scans with radioactive sources and the study of hit efficiency as a function of amplifier threshold. No gain loss is observed after receiving $1.3 \mathrm{fb}^{-1}$ of integrated luminosity corresponding to an integrated charge of $0.055 \mathrm{C} / \mathrm{cm}$ in the hottest spot of the detector.
\end{abstract}

Keywords: Gas detectors, Ageing, Gain loss, Hit efficiency

\section{The LHCb Outer Tracker}

The $\mathrm{LHCb}$ experiment is a single arm spectrometer, located at the LHC, designed to measure $\mathrm{CP}$ violation and rare $B$ decays. The tracking system consists of silicon strip detectors and straw-tube detectors around a large dipole magnet. The

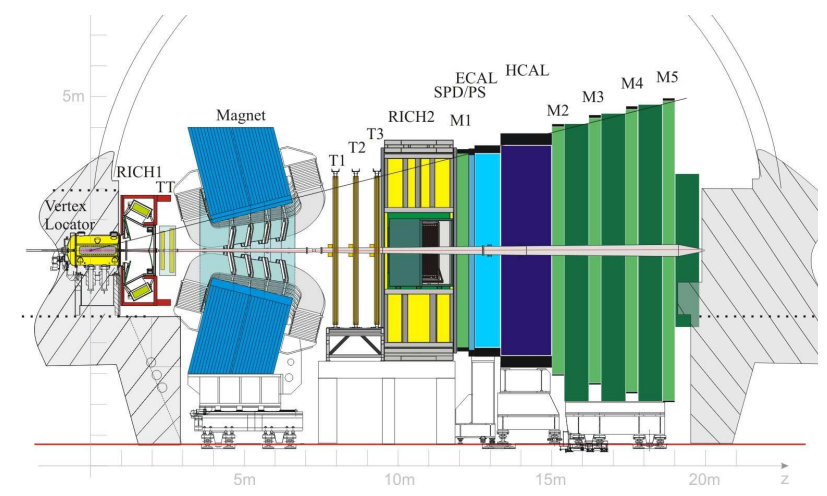

Figure 1: Schematic picture of the LHCb experiment and all its subdetectors. The interaction point is located in the Vertex Locator on the left. The OT consist of three tracking stations indicated by T1, T2 and T3 and covers the region behind the magnet.

\footnotetext{
* Corresponding author

Email address: dveijk@nikhef .nl (D. van Eijk)
}

large area behind the magnet is covered by the Outer Tracker (OT) detector, as indicated in Fig. 1. The OT is a gaseous strawtube detector [1] consisting of 53760 straw tubes and covering an area of approximately $5 \times 6 \mathrm{~m}^{2}$ with 12 detection layers. Every detection layer consists of a double layer of straw tubes as indicated in Fig. 2 (a).

The straw tubes are $2.4 \mathrm{~m}$ long and $4.9 \mathrm{~mm}$ in diameter, and are filled with the gas mixture $\mathrm{Ar} / \mathrm{CO}_{2} / \mathrm{O}_{2}(70 \% / 28.5 \% / 1.5 \%)$ at an exchange rate of about 0.2 volumes per hour. The $\mathrm{O}_{2}$ component is added to the gas mixture for its beneficial effect on the ageing rate [2]. A high voltage of $1550 \mathrm{~V}$ is applied to the anode wire, corresponding to a gain of about $5 \times 10^{4}$ [3]. The anode is made of gold-plated tungsten wire of $25 \mu \mathrm{m}$ diameter, whereas the cathode consists of an inner foil of electrically

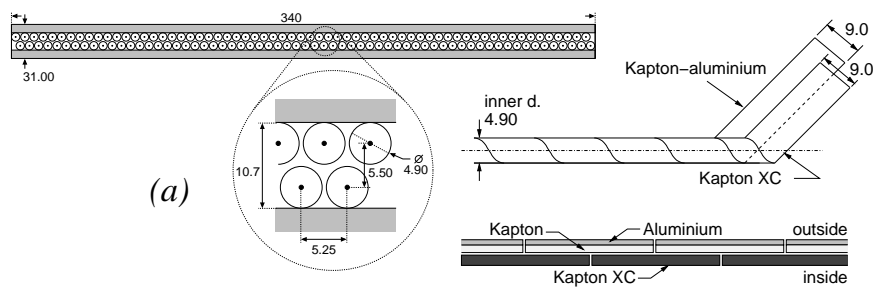

(b)

Figure 2: (a) Cross-section of an OT module containing $2 \times 64$ straw cells. (b) The straws are wound using two foils, Kapton-XC and a laminate of kapton and aluminum. All dimensions are given in $\mathrm{mm}$. 
conducting carbon doped Kapton- $\mathrm{XC}^{1}$ and an outer foil consisting of Kapton-XC laminated with a layer of aluminum. The straws are glued to panels and sealed with sidewalls, resulting in a gas-tight box enclosing a stand-alone detector module. A sketch of the module layout is shown in Fig. 2.

\section{Ageing of OT Modules}

\subsection{Laboratory Tests}

Laboratory tests with radioactive sources revealed that, despite extensive ageing tests in the R\&D phase, the OT modules suffer from gain loss after moderate irradiation (i.e. moderate collected charge per unit time), corresponding to approximately $2 \mathrm{nA} / \mathrm{cm}$. Gain losses of 5-25\% were observed after 20 hours of irradiation. The origin of the gain loss was traced to an insulating layer containing carbon on the anode wire [2], which is caused by glue outgassing components inside the gas volume [4].

The characteristic feature of this ageing phenomenon is a small area of gain loss upstream the radioactive source position. No gain loss is observed downstream the source, presumably due to the formation of ozone in the high intensity region [2]. The contaminated wires have shown to recover the gain after applying a large high voltage of about $1900 \mathrm{~V}$ to the anode wire, inducing large dark currents, or by applying a large high voltage of $1860 \mathrm{~V}$ while irradiating with a radioactive source [4].

\subsection{Conditions During LHC Operation}

During most of the 2011 running period, $\mathrm{LHCb}$ was operating at an instantaneous luminosity of $3.5 \times 10^{32} \mathrm{~cm}^{-2} \mathrm{~s}^{-1}$, which corresponds to a current of $700 \mathrm{nA}$ in the straws located closest to the beam line. The central region of the detector is subject to the largest intensity, corresponding to $14 \mathrm{nA} / \mathrm{cm}$. The LHC delivered a total integrated luminosity of $1.3 \mathrm{fb}^{-1}$ to $\mathrm{LHCb}$ in 2011, which translates in a total accumulated dose at the hottest spot of the OT of $0.055 \mathrm{C} / \mathrm{cm}$.

Two methods to monitor gain stability in the OT will be discussed: scans of the module response with radioactive sources and the study of hit efficiency as a function of amplifier threshold.

\section{Scans with Radioactive Sources}

\subsection{Setup}

The response of OT modules to radioactive sources before and after LHC operation are performed manually in the $\mathrm{LHCb}$ cavern and hence only when the LHC is not in operation. A picture of the setup is shown in Fig. 3 (a). It consists of a frame installed in front of the OT modules accommodating a source holder and a step motor used to move the source holder vertically along the modules. The detector response is determined using two $74 \mathrm{MBq}{ }^{90} \mathrm{Sr}$ sources by measuring the induced current through the wires with a stand-alone current meter. The radiation damage in terms of gain loss is quantified by comparing

\footnotetext{
${ }^{1}$ Kapton® is a polyimide film developed by DuPont.
}

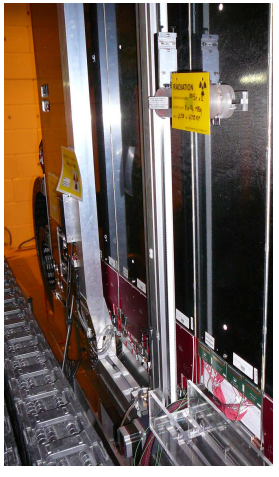

(a)

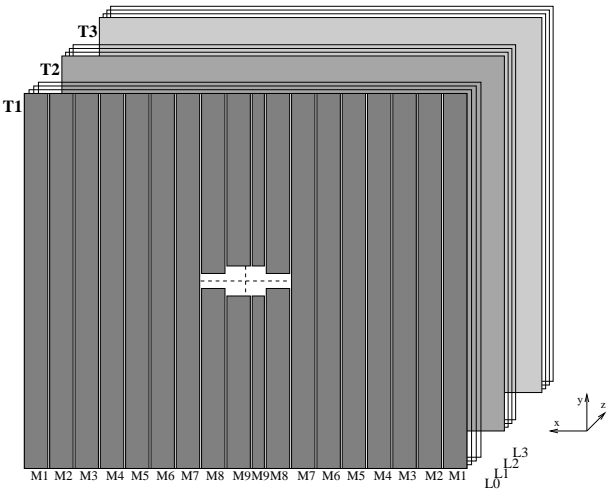

(b)
Figure 3: (a) Picture of the scanning setup. The OT modules are visible, as well as the two ${ }^{90} \mathrm{Sr}$ sources in the source holder, which is mounted on the scanning frame. The front-end electronics at the bottom of the scanned module are replaced by a stand-alone current meter. (b) Schematic picture of the arrangement of the modules in the LHCb detector.

the 2-dimensional current profiles before and after irradiation by LHC operation. The ratio of the currents is expected to be close to unity if no insulating layer is formed on the anode wire.

\subsection{Results}

The lower half of nine modules, corresponding to a quarter of a detector layer (see Fig. 3 (b)) were scanned before and after LHC operation in 2011. The current variation in one wire as a function of the source position is shown in Fig. 4(a). The ratio of currents for all 64 wires in one monolayer and for all positions is shown in Fig. 4(b). The variations in the relative detector response of $\pm 10 \%$ over the width of the module are attributed to small changes in the source profile between the two scans.

The average change in detector response between January 2011 and December 2011 amounts to -3\%, after correcting for changes in the atmospheric pressure. This is mainly attributed to the natural decay of ${ }^{90} \mathrm{Sr}$, which results in a lower detector current of about $3 \%$ after 1 year.

\subsection{Curing}

Before LHC operation, in February 2009, the radiation hardness of the OT modules was studied by irradiating a module with a single $74 \mathrm{MBq}{ }^{90} \mathrm{Sr}$ source during 84 hours. The source was collimated with a hole of $6 \mathrm{~mm}$ diameter, resulting in an irradiated area of about $4 \times 4 \mathrm{~cm}^{2}$, with a maximum dose of 0.015 $\mathrm{C} / \mathrm{cm}$. A maximum gain loss of $38 \%$ was observed. The module was scanned again in January 2011 and July 2011 after LHC operation which corresponds to a delivered integrated luminosity of $0.042 \mathrm{fb}^{-1}$ and $0.434 \mathrm{fb}^{-1}$ respectively.

The irradiated area is located $1.2 \mathrm{~m}$ below the beam axis where the intensity induced by the LHC is approximately $0.15 \mathrm{nA} / \mathrm{cm}$. The corresponding total accumulated dose from the LHC in the irradiated area amounts to about $0.2 \mathrm{mC} / \mathrm{cm}$. The irradiated area shows a partial recovery of about $10 \%$ in January 2011 and a complete recovery of the gain in July 2011. 

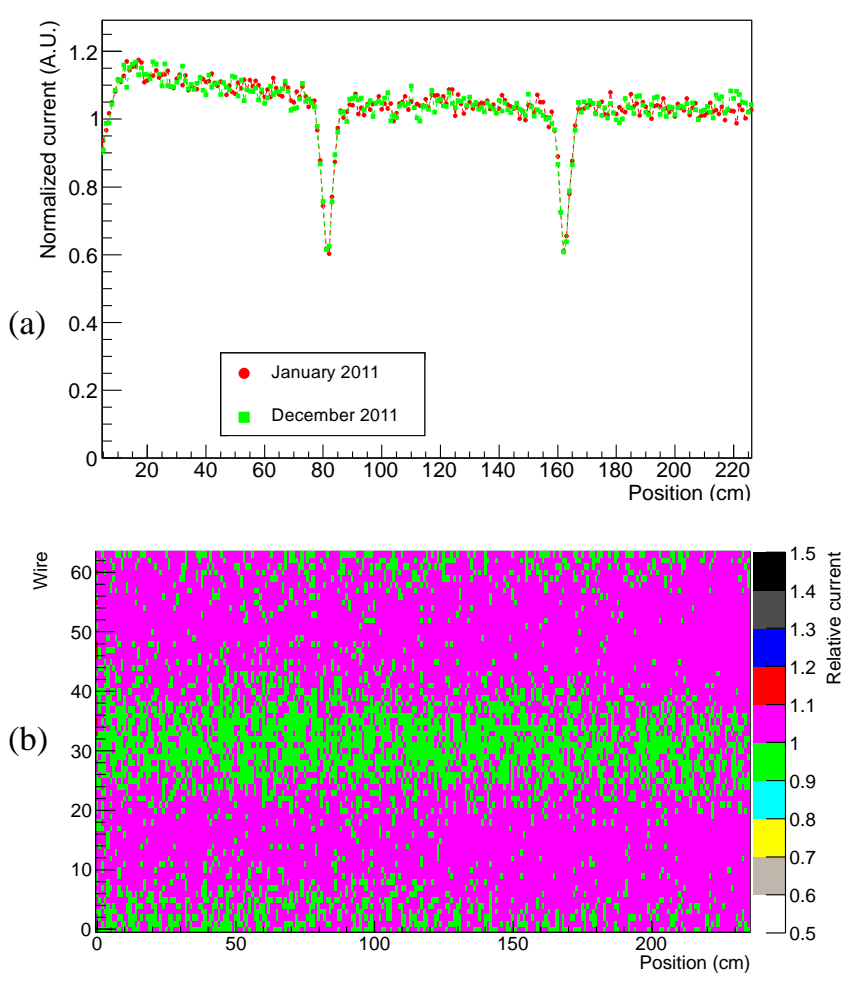

Figure 4: Gain stability scans of an OT module before and after LHC operation in 2011. (a) Normalized current through a wire in January 2011 (red circles) and in December 2011 (open green squares). The current drop at positions $80 \mathrm{~cm}$ and $160 \mathrm{~cm}$ is due to wire locators inside the straw. (b) Relative detector response between January 2011 and December 2011 as a function of wire and position on the module. (For interpretation of the references to color in this figure caption, the reader is referred to the web version of this article.)

The current in one wire of this module is shown in Fig. 5. The observed effect is similar to the curing effect after applying high voltage [4]. It is unclear whether the underlying microscopic mechanism is related to plasma sputtering of the wire surface or to chemical reactions with radicals such as ozone. An attempt was made to reproduce this curing effect in January 2012, but no gain loss could be provoked after 350 hours of irradiation with a single $74 \mathrm{MBq}{ }^{90} \mathrm{Sr}$ source.

\section{Amplifier Threshold Scan}

The scans with radioactive sources can only be performed when the LHC is not operational, and only a small selection of modules can be studied. Therefore, a method to monitor gain stability in the entire OT and during LHC operation was devised. The readout electronics of the OT is designed to accurately determine the time of the hit, but not the charge of the hit. However, by studying the hit efficiency as a function of amplifier threshold during LHC operation, gain variations can be monitored.

\subsection{Method}

The nominal amplifier threshold is $800 \mathrm{mV}$, corresponding to a charge collection of approximately $4 \mathrm{fC}$. The amplifier threshold for a given detection layer is increased in steps from the

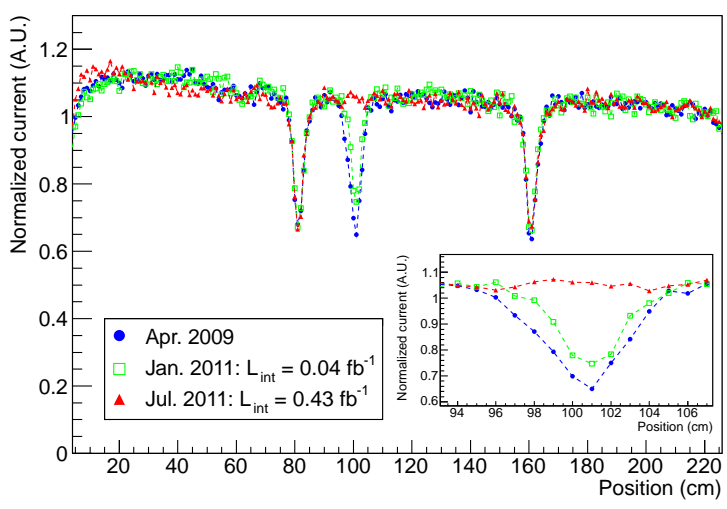

Figure 5: Normalized current as a function of position along a wire in April 2009 (blue circles), January 2011 (open green squares) and July 2011 (red triangles). The current loss around position $100 \mathrm{~cm}$ in April 2009 is caused by a deliberate irradiation (inset: zoom of irradiated area). This loss is partly recovered in January 2011 due to LHC operation in 2010 and fully recovered in July 2011 after an additional six months of LHC operation. The current drop at positions $80 \mathrm{~cm}$ and $160 \mathrm{~cm}$ is due to wire locators in the module. (For interpretation of the references to color in this figure caption, the reader is referred to the web version of this article.)

nominal value of $800 \mathrm{mV}$ to $1450 \mathrm{mV}$ for one OT layer, while all other layers are operated at nominal threshold, in order to properly reconstruct charged particle tracks. This procedure is repeated for all 12 layers.

The hit efficiency is determined using tracks with at least 20 hits in the layers operated at nominal threshold. The hit efficiency is defined as the number of found hits, divided by the total number of predicted hits, for tracks passing within $1.25 \mathrm{~mm}$ from the wire. The hit efficiency is measured in $85 \mathrm{~mm}$ wide bins of the horizontal coordinate $x$ and $56 \mathrm{~mm}$ high bins of the vertical coordinate $y$. The bin size in $x$ corresponds to one quarter of the width of an OT module.

The hit efficiency as a function of amplifier threshold is shown in Fig. 6. This characteristic S-curve can be parameterized using the error function $\operatorname{erf}(x)$ as:

$$
\epsilon_{\mathrm{hit}}\left(V_{\mathrm{thr}}\right)=\frac{1}{2}(P+T)-\frac{1}{2}(P-T) \operatorname{erf}\left(\frac{V_{\mathrm{thr}}-H}{\sqrt{2} \sigma}\right) .
$$

The parameters $P$ and $T$ describe the plateau and the tail of the S-curve respectively. The parameter $H$ is the so-called halfefficiency point, the amplifier threshold at which the efficiency has dropped to $\frac{1}{2}(P+T)$, while $\sigma$ accounts for noise.

Initially, only eight threshold steps per layer were recorded. Since the hit efficiency was poorly constrained in the tail, two points at $1600 \mathrm{mV}$ and $1800 \mathrm{mV}$ were added in the threshold scans recorded from June 2011 onwards. The hit efficiency at $1600 \mathrm{mV}$ and $1800 \mathrm{mV}$ is observed to be essentially zero and therefore the tail parameter $T$ is fixed to zero in the S-curve fit. For a fair comparison between S-curve fits in different threshold scans, only the eight measurements below $1600 \mathrm{mV}$ are taken into account in the fit. The result of the fit of Eq. 1 to the hit efficiency as a function of the threshold is shown by the continuous curve in Fig. 6.

Ageing in the OT would reduce the charge amplification, due to the insulating layer on the anode wires. This would lead to 


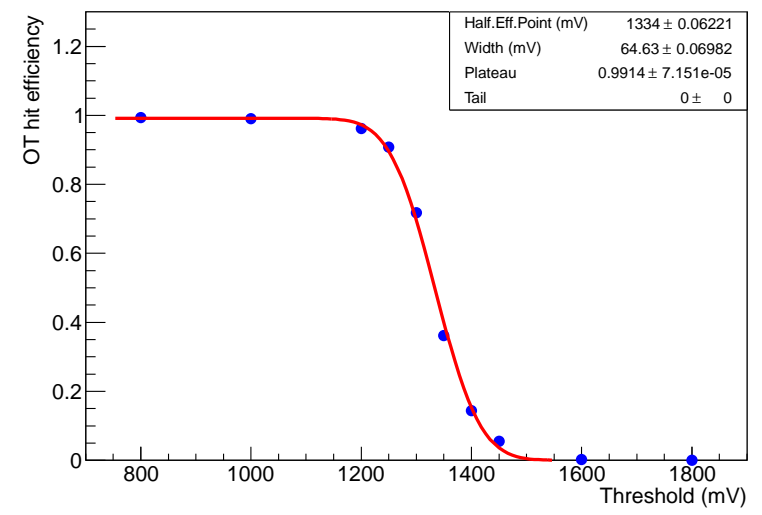

Figure 6: S-curve fit to data from a threshold scan recorded in October 2011 , for the OT detection layer closest to the interaction point. The tail $T$ is fixed to $0, P$ is found to be 0.99 and $H$ is fitted as $1334 \mathrm{mV}$. Notice that the two points at $1600 \mathrm{mV}$ and $1800 \mathrm{mV}$ are not taken into account in the fit, for fair comparison between all threshold scans.

an S-curve shifted to lower values of the amplifier threshold, resulting in a smaller half-efficiency point. The stability of the half-efficiency point between threshold scans is used to monitor gain variations in any layer and at any position in $x$ and $y$. The threshold scans are performed on a regular basis, such that possible ageing in the OT can be detected at an early stage, before the hit efficiency under nominal conditions is affected.

\subsection{Gain Variations in the $O T$}

To relate shifts in half-efficiency point to gain variations, the shift in $H$ as a function of high voltage was measured [3, 5]. Since the relation between gain and $\mathrm{HV}$ is known, the shift in half-efficiency point $\Delta H=H_{2}-H_{1}$ as a function of the relative

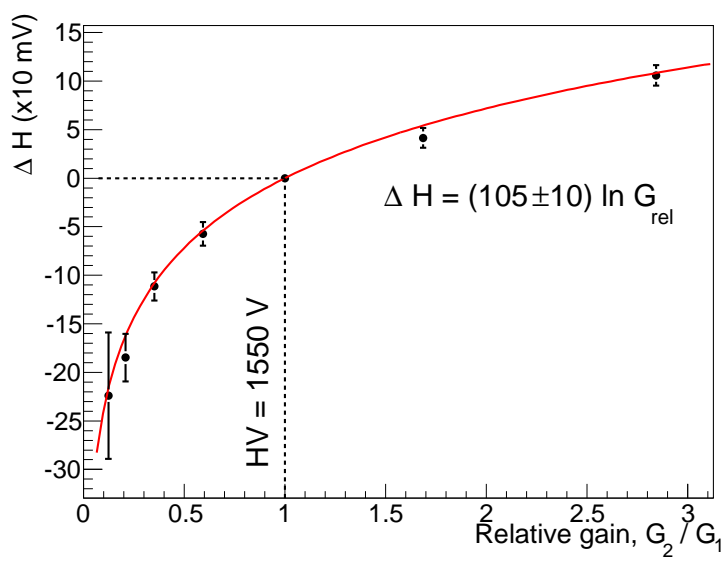

Figure 7: Calibration curve of the shift in half-efficiency point $H$ versus the relative gain. The data points and uncertainties are obtained from the average shift in $\mathrm{H}$ of the 64 wires in the module under test.

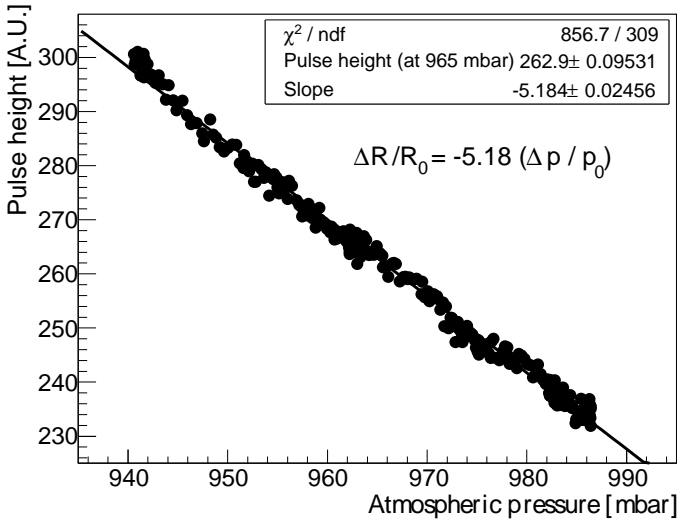

Figure 8: Pulse height, $R$, versus atmospheric pressure, $p$, as measured on a test module in the LHCb cavern.

gain $G_{\mathrm{rel}}=\frac{G_{2}}{G_{1}}$, was determined, and parameterized as (Fig. 7):

$$
G_{\mathrm{rel}}=\exp \left(\frac{\Delta H[\mathrm{mV}]}{105 \mathrm{mV}}\right) .
$$

A correction for the atmospheric pressure $p$ is determined from the pulse height $(R)$ variation as a function of atmospheric pressure shown in Fig. 8, which is obtained from a dedicated test module which is constantly irradiated by a radioactive ${ }^{55} \mathrm{Fe}$ source. Since gain is proportional to pulse height the relative gain is equal to the relative pulse height, which is found to be

$$
\frac{\Delta G}{G}=\frac{\Delta R}{R_{0}}=-5.18 \frac{\Delta p}{p_{0}} .
$$

\subsection{Threshold Scan Results}

Throughout the 2010 and 2011 run periods, OT threshold scans were performed at regular intervals, corresponding to about $200 \mathrm{pb}^{-1}$ of delivered integrated luminosity. The duration of one threshold scan is approximately one hour, collecting about $1.5 \times 10^{5}$ events (corresponding to roughly $3 \times 10^{6} \operatorname{good}$ quality tracks) at each threshold setting.

The half-efficiency point $H$ is obtained from a fit of the $\mathrm{S}$ curve in every bin, as parameterized in Eq. 1, and is shown in bins of $x$ and $y$ in Fig. 9 for two threshold scans. The first scan is recorded in August 2010, before nominal LHC operation and the second scan is recorded in October 2011.

The values for $H$ in every bin from the scans in August 2010 and October 2011 are subtracted, and the relative gain per bin is calculated using the calibration of Eq. 2 and corrected for the atmospheric pressure. The pressure-corrected relative gain per bin in $x$ and $y$ is shown in Fig. 10. Apart from bin-to-bin fluctuations, no areas with gain loss (relative gain smaller than 1) are observed. The statistical accuracy degrades towards the edges of the OT resulting in larger bin-to-bin fluctuations.

To increase sensitivity, the hit efficiency is averaged over regions of the OT in $x$ and $y$. Six regions in $(x, y)$ coordinates are studied, averaged over all 12 layers. The inner region is defined as the region within $\pm 60 \mathrm{~cm}$ in both $x$ and $y$ from the beam pipe 

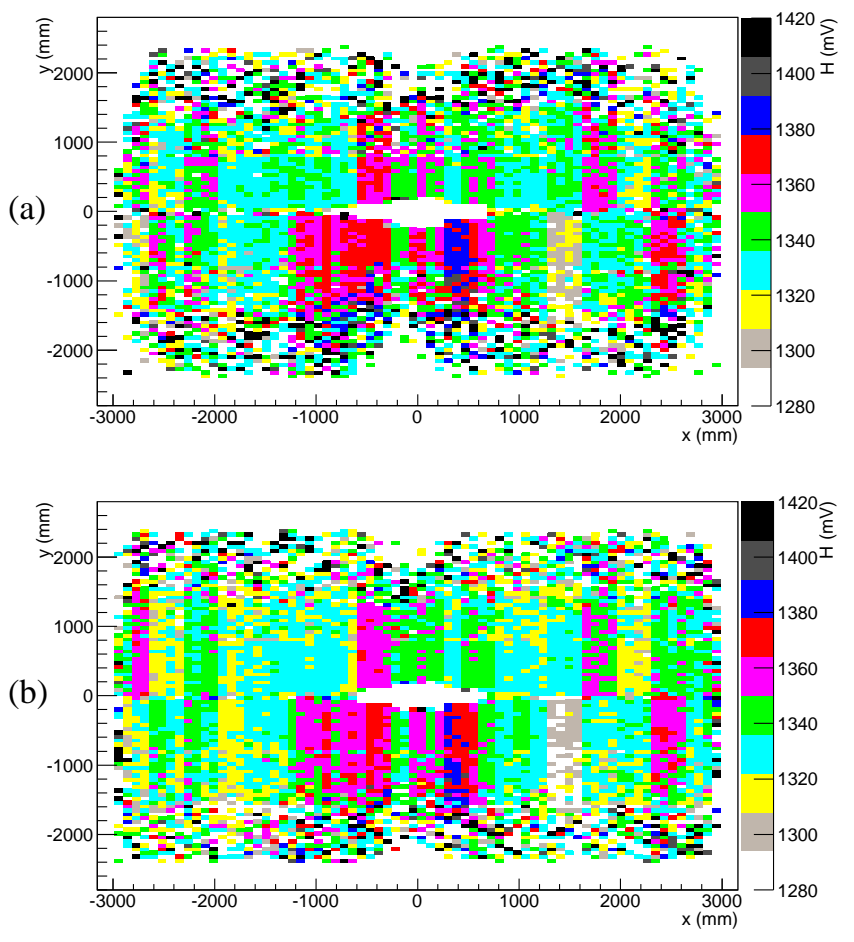

Figure 9: Fitted half-efficiency point $H$ in $m V$ as a function of $x$ and $y$ for $O T$ layer 8 in August 2010 (a) and October 2011 (b). Differences in threshold characteristics of the individual readout electronics units result in the observed module-to-module variations.

and is subject to the highest particle intensity. The outer region is the region outside $\pm 60 \mathrm{~cm}$ in $x$ and $y$ from the beam pipe. The lower (upper) region is defined as $y<-60 \mathrm{~cm}(y>60 \mathrm{~cm})$. The region closest to the gas inlet and outlet are defined as $y<-200 \mathrm{~cm}$ and $y>200 \mathrm{~cm}$, respectively.

As an example, the S-curves for the inner region are shown in Fig. 11. The shift in fitted half-efficiency point between the two S-curves is $\Delta H=-3.7 \mathrm{mV}$, with a negligible statistical uncertainty, which corresponds to an uncorrected relative gain of 0.965. Correcting for atmospheric pressure differences during the two scans, $975.0 \mathrm{hPa}$ and $985.3 \mathrm{hPa}$ in August 2010 and

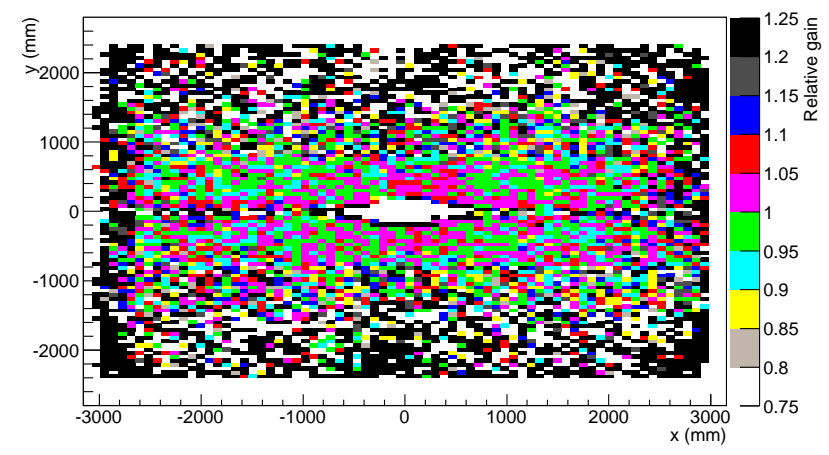

Figure 10: Pressure-corrected relative gain in bins of $x$ and $y$ for layer 8 of the OT between October 2011 and August 2010. (a)

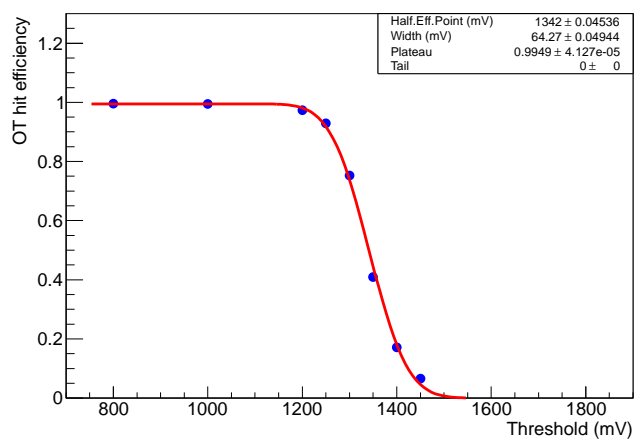

(b)

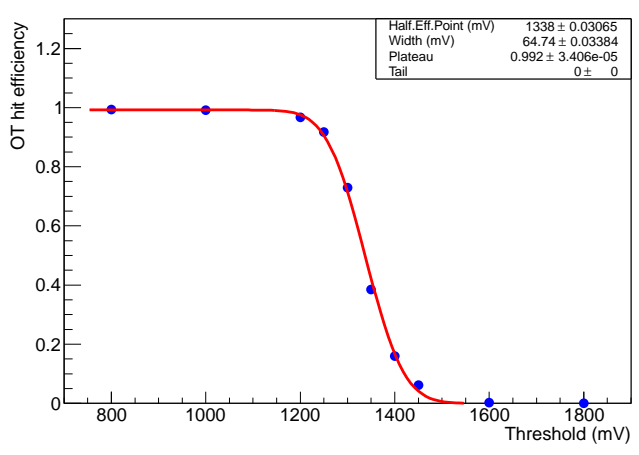

Figure 11: S-curve for August 2010 (a) and October 2011 (b) for the inner region, defined as $\pm 60 \mathrm{~cm}$ in $x$ and $\pm 60 \mathrm{~cm}$ in y from the central beam pipe, summed over all OT layers. (Notice that the threshold scan in October 2011 contains two extra data points up to $1800 \mathrm{mV}$ which are not used in the fit for fair comparison between threshold scans.)

October 2011 respectively, this number changes to 1.021 . The pressure-corrected relative gain variation is thus $+2.1 \%$ for the inner region from August 2010 to October 2011. The results for the other regions, integrated over the entire OT, are presented in Table 1, showing a uniform response over the OT surface.

\begin{tabular}{|c|c|c|}
\hline region & coordinates $\left(x_{1}, x_{2}\right),\left(y_{1}, y_{2}\right)(\mathrm{cm})$ & $\begin{array}{c}\text { pressure-corrected } \\
\text { relative gain variation }\end{array}$ \\
\hline \hline entire OT & $(-300,300)(-250,250)$ & $+1.3 \%$ \\
\hline inner & $(-60,60)(-60,60)$ & $+2.1 \%$ \\
\hline outer & outside of $(-60,60)(-60,60)$ & $+0.9 \%$ \\
\hline lower & $(-300,300)(60,250)$ & $-0.2 \%$ \\
\hline upper & $(-300,300)(-250,-60)$ & $+0.2 \%$ \\
\hline gas inlet & $(-300,300)(200,250)$ & $+0.8 \%$ \\
\hline gas outlet & $(-300,300)(-250,-200)$ & $-2.0 \%$ \\
\hline
\end{tabular}

Table 1: Relative gain variation between August 2010 and October 2011 for various OT regions, summed over all 12 layers and corrected for the change in atmospheric pressure. The various regions are indicated by their coordinates in $x$ and $y$.

\subsection{Systematic Uncertainties}

To estimate the systematic uncertainty of the method, the fit and the comparison procedures have been varied. For every systematic change, the analysis of the scans in August 2010 and October 2011 is repeated for all regions and the largest deviation in relative gain variation with respect to the nominal analysis is taken as the systematic uncertainty. 


\begin{tabular}{|c|c|}
\hline systematic check & $\begin{array}{c}\text { largest difference in relative } \\
\text { gain variation per region }\end{array}$ \\
\hline \hline$T$ free & $+1.2 \%$ \\
\hline fix $P=1$ & $\pm 0.0 \%$ \\
\hline pressure correction & $+0.4 \%$ \\
\hline calibration curve $\pm 1 \sigma$ & ${ }_{-0.8 \%}^{+0.8 \%}$ \\
\hline definition $H$ & $-0.4 \%$ \\
\hline double Gaussian fit & $+1.5 \%$ \\
\hline \hline total & $\pm 2.2 \%$ \\
\hline
\end{tabular}

Table 2: Changes to the fit and to the scan comparisons were applied to estimate the systematic uncertainty. The right column shows the largest deviation in relative gain variation from the nominal analysis in the various regions. The total systematic uncertainty is the quadratic sum.

The first check is to float the value of the tail parameter $T$ in the fit. A second check is to constrain $P=1$, in addition to $T=0$. Subsequently, the correction for the atmospheric pressure is varied by a relative $\pm 10 \%$. The fitted parameter of the calibration curve of $\Delta H$ versus relative gain was varied by $\pm 1 \sigma$ and the biggest difference is assigned as systematic error. In addition, the definition of $H$ is changed to the threshold at which the hit efficiency is 0.5 instead of $\frac{1}{2}(P+T)$.

The largest difference in relative gain variation per region for each systematic check is summarized in Table 2. The systematic uncertainties of all checks are added in quadrature and a total systematic uncertainty of $2.2 \%$ is assigned to the method.

\subsection{Time Trend of Relative Gain Variation}

In total, eight full threshold scans have been recorded in 2010 and 2011. Using the scan from August 2010 as a reference, the relative gain variation as a function of date and as a function of delivered integrated luminosity, averaged over the entire OT is shown in Fig. 12 (a) and (b), respectively.

The observed gain increase for the scan in October 2010 with respect to August 2010 (corresponding to a delivered integrated luminosity of $0.031 \mathrm{fb}^{-1}$ ) is not well understood. Overall relative gain variations could be due to variations in the gas mixture. However, the gas mixture is controlled at a level nominally better than $0.2 \%$, which would result in a maximum gain variation of $2 \%$ and hence could not explain the observed change in detector response. Relative gain variations could also be caused by different run conditions. For example, the average number of $p p$ interactions per bunch crossing is directly correlated to the event occupancy, which influences the hit efficiency. However, no relation is found between run conditions and the observed relative gain variations. For the scans taken after October 2010, no significant time dependence is observed.

\section{Conclusion}

Gain stability in the LHCb Outer Tracker is monitored using two techniques: scanning OT modules with a radioactive source and studying hit efficiency as a function of amplifier threshold. The first method compares the module response to ${ }^{90} \mathrm{Sr}$ (a)

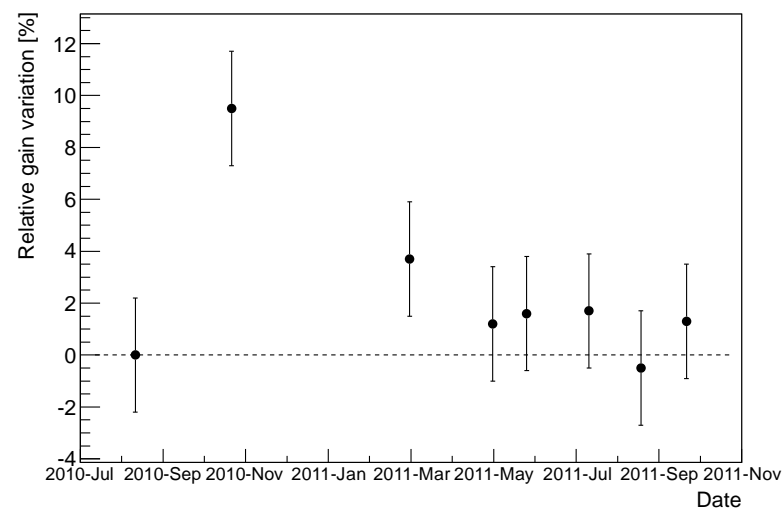

(b)

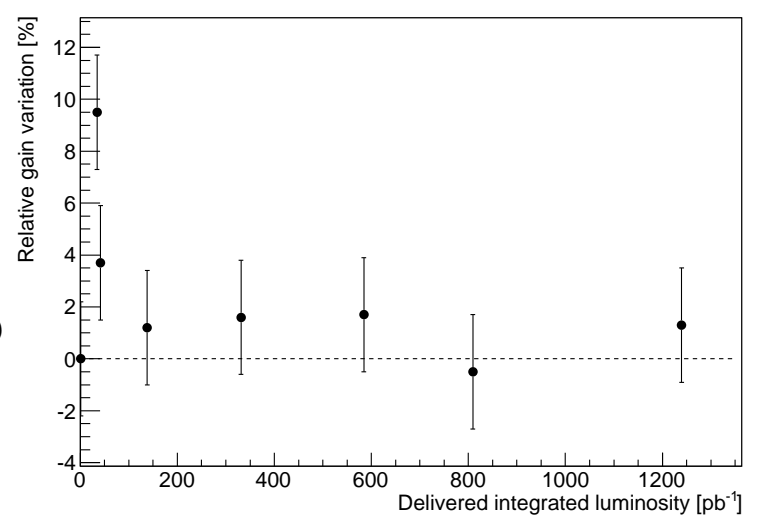

Figure 12: Relative gain variation averaged over the entire OT, compared to August 2010 (indicated by the dashed line) versus date (a) and versus delivered integrated luminosity $(b)$. The error bars indicate the total systematic uncertainty from Table 2 and are fully correlated between the points.

sources and can only be applied to a small set of modules in periods in which the LHC is not operational. No significant gain loss (about $-3 \%$ ) is observed in the ${ }^{90} \mathrm{Sr}$ scans between January 2011 and December 2011, which is attributed to the decrease of the source strength. The second technique uses the OT readout electronics to study hit efficiency as a function of amplifier threshold during LHC operation. Using this method, the relative gain variation averaged over the entire OT between August 2010 and October 2011 is $(+1.3 \pm 2.2) \%$. This indicates that no gain loss is observed in the OT after LHC operation in 2010 and 2011 .

\section{Acknowledgments}

We would like to thank Roel Aaij, Johannes Albrecht and Gerhard Raven for their help on the trigger configuration used in the threshold scans. In addition we would like to thank Rainer Schwemmer and Richard Jacobsson for their help in recording the threshold scans in a proper way and for providing additional information on run conditions during the threshold scans. 


\section{References}

[1] LHCb Coll., The LHCb Detector at the LHC, Journal of Instrumentation 3 (2008) S08005.

[2] S. Bachmann et al., Ageing in the LHCb Outer Tracker: Phenomenon, Culprit and Effect of Oxygen, Nuclear Instruments and Methods A 617 (2010) 202.

[3] G. Apeldoorn et al., Avalanche and streamer production in $\mathrm{Ar} / \mathrm{CO}_{2}$ mixtures, LHCb-2005-038, 2005.

[4] N. Tuning et al., Ageing in the LHCb outer tracker: Aromatic hydrocarbons and wire cleaning, Nuclear Instruments and Methods A 656 (2011) 45.

[5] E.L. Visser, Preventing, monitoring and curing the ageing in the $\mathrm{LHCb}$ Outer Tracker, CERN-THESIS-2010-094, 2010. 